\title{
SYMMETRY FOR FINITE DIMENSIONAL HOPF ALGEBRAS
}

\author{
J. E. HUMPHREYS ${ }^{1}$
}

\begin{abstract}
This note refines criteria given by $\mathbf{R}$. G. Larson and M. E. Sweedler for a finite dimensional Hopf algebra to be a symmetric algebra, with applications to restricted universal enveloping algebras and to certain finite dimensional subalgebras of the hyperalgebra of a semisimple algebraic group in characteristic $p$.
\end{abstract}

Let $A$ be a finite dimensional associative algebra over a field $K$. Then $A$ is called Frobenius if there exists a nondegenerate bilinear form $f: A \times A \rightarrow K$ which is associative in the sense that $f(a b, c)=f(a, b c)$ for all $a, b, c \in A[3$, Chapter IX]. $A$ is called symmetric if there exists a symmetric form of this type $[3, \S 66]$. For example, semisimple algebras and group algebras of finite groups are symmetric. We investigate here the extent to which finite dimensional Hopf algebras (with antipode) are symmetric; they are always Frobenius, thanks to the main theorem of [8].

1. Hopf algebras. In this section $H$ denotes a finite dimensional Hopf algebra over an arbitrary field $K$, with antipode $s$ and augmentation $\varepsilon$ : $H \rightarrow K$. According to the main theorem of [8], existence of the antipode implies (and is implied by) the existence of a (nonsingular) left integral $\Lambda \in H$, which is unique up to scalar multiples. By definition, $\Lambda$ satisfies: $h \Lambda=\varepsilon(h) \Lambda$, for all $h \in H$. Equally well, $H$ has a right integral $\Lambda^{\prime}$, unique up to scalar multiples. If $\Lambda^{\prime}$ is proportional to $\Lambda, H$ is called unimodular.

With a left integral $\Lambda$ is associated a nondegenerate bilinear associative form $b$ on $H[8, \S 7]$. As a result, $H$ is a Frobenius algebra. From the second corollary of Proposition 8 in [8], applied to the dual Hopf algebra (whose antipode has the same order as $s$ ), we obtain immediately:

THEOREM 1. With notation as above, $b$ is symmetric if and only if $H$ is unimodular and $s^{2}=1$. In particular, if the latter conditions hold, then $H$ is symmetric.

We can apply this to the algebras $u_{n}(n=1,2, \ldots)$ defined in [6, Appendix U], [7]. These are finite dimensional Hopf subalgebras of the hyperalgebra $U_{K}$ of a simply connected, semisimple algebraic group $G$ over an algebraically

Received by the editors February 28, 1977.

AMS (MOS) subject classifications (1970). Primary 16A24, 16A36, 17B50; Secondary 17B45.

Key words and phrases. Hopf algebra, Frobenius algebra, symmetric algebra, restricted Lie algebra, integral, unimodular, hyperalgebra of semisimple algebraic group.

${ }^{1}$ Research partially supported by NSF Grant MCS72-05055 A04. 
closed field $K$ of characteristic $p>0$, with antipode of order 2 .

COROLlaRY. The algebras $u_{n}$ are symmetric.

Proof. $G$ acts naturally on $u_{n}$ as a group of Hopf algebra automorphisms (adjoint action) and therefore $g \Lambda$ is another left integral, if $g \in G$ and $\Lambda$ is a left integral in $u_{n}$; this must be of the form $\alpha(g) \Lambda$ for $\alpha(g) \in K^{*}$. Since $G=(G, G)$, it has no nontrivial homomorphisms into $K^{*}$, forcing $\alpha=1$; therefore, $\Lambda$ is $G$-invariant. In turn, $\Lambda$ is invariant under the derived adjoint action of $u_{n}$, i.e., $\Lambda$ lies in the center of $u_{n}$ and is therefore also a right integral. Now $u_{n}$ is unimodular, with antipode of order 2, so the theorem applies. Q.E.D.

Remarks. (1) W. J. Haboush [4] has recently been able to construct explicitly an integral for each $u_{n}$, and has used these to obtain a new family of "central differential operators" which are capable of separating the various Steinberg modules for $G$. His work inspired the present note, in particular the proof of the corollary.

(2) It follows from the corollary that the Cartan matrix $C$ of $u_{n}$, recording the composition factor multiplicities of principal indecomposable modules, is symmetric. Perhaps it is even of the form $C={ }^{t} D \cdot D$ for a suitable "decomposition matrix" $D$. This is the case when $n=1$ [5], $u_{1}$ being the restricted universal enveloping algebra of the Lie algebra of $G$.

THEOREM 2. If $H$ is symmetric, then $H$ is unimodular.

Proof. Let $f$ be a nondegenerate symmetric, associative bilinear form on $H$, and let $\Lambda$ (resp. $\Lambda^{\prime}$ ) be a left (resp. right) integral. If $h \in \operatorname{Ker} \varepsilon, f(h, \Lambda)=$ $f(1, h \Lambda)=0=f\left(\Lambda^{\prime} h, 1\right)=f\left(\Lambda^{\prime}, h\right)$. Since $f$ is symmetric, both $\Lambda$ and $\Lambda^{\prime}$ lie in the orthogonal complement of $\operatorname{Ker} \varepsilon$, which has dimension 1 . So $\Lambda$ is proportional to $\Lambda^{\prime}$. Q.E.D.

If $H$ is commutative or cocommutative, then automatically $s^{2}=1$ (cf. [8, p. 77]); in any event, $s$ has finite order [9]. But examples are known for which $s$ has higher (necessarily even) order. It is not clear whether such algebras can be unimodular without being symmetric.

2. Restricted enveloping algebras. In this section $K$ is a field of characteristic $p>0, L$ a restricted Lie algebra (Lie $p$-algebra) over $K, u(L)$ its restricted universal enveloping algebra, which is a finite dimensional Hopf algebra with antipode of order 1 or 2. A. Berkson [1] showed directly that $u(L)$ is Frobenius by using a bilinear form $f$ defined as follows. Fix an ordered basis $x_{1}, \ldots, x_{n}$ of $L$, so the monomials $x_{1}^{i_{1}} \cdots x_{n}^{i_{n}}\left(0 \leqslant i_{j}<p\right)$ form a basis of $u(L)$. Let $\varphi_{0}$ be the linear function taking value 1 at $u_{0}=x_{1}^{p-1} \cdots x_{n}^{p-1}$ and value 0 at other monomials, and define $f(u, v)=\varphi_{0}(u v)$. In turn, J. R. Schue proved:

THEOREM 3 [10]. The bilinear form $f$ is symmetric if and only if $\operatorname{Tr}(\operatorname{ad} x)=0$ 
for all $x \in L$. In particular, if the latter condition is satisfied, $u(L)$ is symmetric.

Here ad $x(y)=[x y]$; the notation $D_{x}$ is used in [10] and $D(x)$ in [8]. This criterion for symmetry was applied in [5] to show that the algebra $u_{1}$ discussed above is symmetric. On the other hand, an example given in [8, p. 85 ] to show that $u(L)$ need not be unimodular also fails (as it must, by Theorems 2 and 3) to meet Schue's criterion.

The third corollary of Proposition 8 in [8] states that $u(L)$ is unimodular if and only if $\operatorname{Tr}(\operatorname{ad} x)=0$ for all $x \in L$. We offer here a different proof, based on the following lemma.

LEMMA. Let $\Lambda$ be a left integral in $u(L)$. If $x_{1}, \ldots, x_{n}$ is any ordered basis of $L$, and $\Lambda$ is written as a linear combination of the monomials $x_{1}^{i_{1}} \cdots x_{n}^{i_{n}}$, then $u_{0}=x_{1}^{p-1} \cdots x_{n}^{p-1}$ must occur with nonzero coefficient.

Proof. Suppose the contrary. Define the degree of $x_{1}^{i_{1}} \cdots x_{n}^{i_{n}}$ to be $\sum i_{j}$, and write $\Lambda=\Lambda_{1}+\Lambda_{2}$, where $\Lambda_{1}$ contains all monomials of the highest degree $d$ occurring (so $d<n(p-1)$ ). If we change basis by permuting the $x_{i}$, the new expression for $\Lambda$ will be of the form $\Lambda_{1}^{\prime}+\Lambda_{3}$, where $\Lambda_{1}^{\prime}$ is obtained from $\Lambda_{1}$ by permuting the $x_{i}$ and the monomials in $\Lambda_{3}$ are again of degree $<d$ (cf. the proof of Lemma 1 in [10]). We may therefore assume that not all monomials occurring in $\Lambda_{1}$ involve the factor $x_{1}^{p-1}$.

The augmentation for $u(L)$ maps all $x_{i}$ to 0 , so by definition of left integral, $0=x_{1} \Lambda=x_{1} \Lambda_{1}+x_{1} \Lambda_{2}$. Because of the $p$-structure, $x_{1}^{p} \in L$, so $x_{1}^{p} x_{2}^{i_{2}} \cdots x_{n}^{i_{n}}$ can be rewritten in $u(L)$ as a linear combination of monomials having degrees $<p+\sum i_{j}$ (sum over $j \neq 1$ ). In particular, $x_{1} \Lambda_{2}$ involves only monomials of degree $\leqslant d$, while $x_{1} \Lambda_{1}$ involves such monomials along with one or more linearly independent monomials of degree $d+1$ (corresponding to monomials in $\Lambda_{1}$ not involving $x_{1}^{p-1}$ ). This is clearly impossible. Q.E.D.

We remark that the integral constructed by Haboush [4] for the algebra $u_{1}$ illustrates this lemma very nicely.

THEOREM 4. If $u(L)$ is a symmetric algebra, then $\operatorname{Tr}(\operatorname{ad} x)=0$ for all $x \in L$.

Proof. Since $u(L)$ is symmetric, it is unimodular (Theorem 2), so any integral $\Lambda$ lies in the center of $u(L)$. Choose an ordered basis $x_{1}, \ldots, x_{n}$ of $L$, and define the form $f$ as above, relative to the basis of $u(L)$ consisting of monomials. By the lemma, we may (after multiplying $\Lambda$ by a nonzero scalar) write $\Lambda=u_{0}+u_{1}$, where $u_{1}$ is a linear combination of monomials of degrees $<n(p-1)$. With $\varphi_{0}$ as above, it follows from Lemma 1 of [10] (cf. proof of theorem) that $\varphi_{0}\left(u_{1} x\right)=\varphi_{0}\left(x u_{1}\right)$ for all $x \in L$. On the other hand, Lemma 3 of [10] says that $\varphi_{0}\left(u_{0} x\right)=\varphi_{0}\left(x u_{0}\right)+\operatorname{Tr}(\operatorname{ad} x)$ for $x \in L$. Since $\Lambda x=x \Lambda$, it follows at once that $\operatorname{Tr}(\operatorname{ad} x)=0$. Q.E.D.

Theorems 3 and 4, combined with Theorems 1 and 2, show that $u(L)$ is unimodular if and only if $\operatorname{Tr}(\operatorname{ad} x)=0$ for all $x \in L$, as stated in [8]. This is analogous to the classical criterion for a Lie group $G$ to be unimodular: 
$\operatorname{det}(\operatorname{Ad} g)=1$ for all $g \in G$ [2, Chapter III, 3, no. 16, Corollary to Proposition 55].

\section{REFERENCES}

1. A. Berkson, The u-algebra of a restricted Lie algebra is Frobenius, Proc. Amer. Math. Soc. 15 (1964), 14-15. MR 28 \#2132.

2. N. Bourbaki, Groupes et algèbres de Lie, Chapters II-III, Hermann, Paris, 1972.

3. C. W. Curtis and I. Reiner, Representation theory of finite groups and associative algebras, Interscience, New York, 1962. MR 26 \#2519.

4. W. J. Haboush, (manuscript).

5. J. E. Humphreys, Modular representations of classical Lie algebras and semisimple groups, J. Algebra 19 (1971), 51-79. MR 44 \#271.

6. __ Ordinary and modular representations of Chevalley groups, Lecture Notes in Math., vol. 528, Springer-Verlag, New York and Berlin, 1976.

7. On the hyperalgebra of a semisimple algebraic group, Contributions to Algebra: A Collection of Papers Dedicated to Ellis Kolchin, Academic Press, New York, 1977.

8. R. G. Larson and M. E. Sweedler, An associative orthogonal bilinear form for Hopf algebras, Amer. J. Math. 91 (1969), 75-94. MR 39 \# 1523.

9. D. E. Radford, The order of the antipode of a finite-dimensional Hopf algebra, Amer. J. Math. 98 (1976), 333-355. MR 53 \# 10852.

10. J. R. Schue, Symmetry for the enveloping algebra of a restricted Lie algebra, Proc. Amer. Math. Soc. 16 (1965), 1123-1124. MR 32 \#2515.

Department of Mathematics, University of Massachusetts, Amherst, Massachusetts 01003

School of Mathematics, Institute for Advanced Study, Princeton, New Jersey 08540

Current address: Department of Mathematics, University of Massachusetts, Amherst, Massachusetts 01003 